\title{
Hybrid Bayesian Network in Neural Network based Deep Learning Framework for Detection of Obstructive Sleep Apnea Syndrome
}

\author{
Farah Nadiah Binti Mohamed Farouk, Toni Anwar, Nordin bin Zakaria
}

\begin{abstract}
This study aimed to develop Bayesian Network model integrated with Deep Learning to help doctors diagnose Obstructive Sleep Apnoea Syndrome (OSAS) more holistically and clearly. The results of this research will produce a useful and beneficial clinical workflow for future support in health care. The model will be developed based on the methods of analysis and the quantitative data used to compromise the developing of Hybrid Bayesian Network in Neural Network using Deep Learning Algorithm. The aim of this study was to apply a hybrid model of convolutional neural network (CNN) that could be used during sleep consultation to determine the need for electrocardiography (ECG) signals stimuli for Polysomnography (PSG).
\end{abstract}

Keywords: Convolution neural network (CNN), Obstructive sleep apnoea syndrome (OSAS), Polysomnography (PSG), Electrocardiography (ECG)

\section{INTRODUCTION}

Obstructive sleep apnoea (OSA) is a sleep-related respiratory syndrome causing a frequent transient breathing delay leading to the upper airway narrowing or closure during sleep. This occurs when the muscle relax when resting, inducing loss of soft tissue at the back of the throat and restricting the upper water. This occurs as muscles relax during sleep, creating a breakdown in the soft tissue behind the neck, covering the upper air. This can contribute to a slight change of breathing or to a full delay in sleep for at least a period of 10 seconds. Anyone can be impacted by OSAS, but in some individuals it is more common: male, elderly, middle aged and might be over-weight as well. The disease must be taken strictly in medical care because it is closely associated with certain major diseases such as high blood pressure, stroke, asthma and diabetes.

Revised Manuscript Received on October 30, 2019.

* Correspondence Author

Farah Nadiah binti Mohamed Farouk, Department of Computer \& Sciences, Universiti Teknologi PETRONAS, Tronoh, Perak, Malaysia. Email: farah_17008070@utp.edu.my

Dr. Toni Anwar, Department of Computer \& Sciences, Universiti Teknologi PETRONAS, Tronoh, Perak, Malaysia.

Email: toni.anwar@utp.edu.my

Dr. M Nordin bin Zakaria, Department of Computer \& Sciences, Universiti Teknologi PETRONAS, Tronoh, Perak, Malaysia.

Email: nordinzakaria@utp.edu.my

(c) The Authors. Published by Blue Eyes Intelligence Engineering and Sciences Publication (BEIESP). This is an open access article under the CC BY-NC-ND license (http://creativecommons.org/licenses/by-nc-nd/4.0/)
The surveillance of OSAS in patients affected, including patients who receive medical therapies or certain types of drugs is essential. In perioperative situations when a patient is undergoing surgery and after that control is extremely important. Presently, clinicians were exposed to a sleep study called Polysomnography (PSG) to evaluate OSAS. Nonetheless, it is quite complex, as it requires electrooculogram, electroencephalogram, oronasal airflow, chest wall and abdominal wall movement, oxygen saturation and Electrocardiogram (ECG) tests. Its procedure has several inconveniences. An added downside, because of the maximum amount of wires, leads, tubes and. Much more connected to the patient, is that patients should remain at the same location during the test for the remainder of the day. There are also no areas to check, implying that clinicians have a long time to wait. A prototype of contactless multisensor has been developed by Promprapa, A., Sayani, M.S., Anwar, T. and others to detect vital parameters and a deep learning hybrid model proposed based on convolutional neural network (CNN) and recurrent neural network (RNN). The study aimed to develop Bayesian Network model integrated with Neural Network to help doctors in getting more holistic and clear diagnosis of OSAS. The findings from this research will produce a useful and benefit medical workflow for future healthcare support. The model will be created based on the research methodology and qualitative data used which compromises every step in developing a Hybrid Bayesian Network- Deep Learning. Few case studies has been chosen in order to define the problem of current Deep Learning System.

\section{OBSTRUCTIVE SLEEP APNEA}

The literature notes that the OSAS treatment tolerance rates in the Health Center are low, as the condition has not been taken seriously [2]. That is because, [2] has proved in their research paper that in United Kingdom, just 58\% people of snore or whose spouse snores in the context of OSAS, $42 \%$ of people did not know about OSAS. [12] believed that OSAS is a very major disease and that it needs to be taken seriously by medical centre, as OSAS impacts not only the person themselves but also family surrounding them. [3] stated that OSAS patient had excessive sleepiness during their day and less professional performance, the pace of response was sluggish, incapable of deciding and also decreased focus. Taking into account the complication of OSAS in brain and cardiovascular system, [12] notes that OSAS could adversely affect line span and quality, which is thought to involve a body multisystem. 


\section{Hybrid Bayesian Network in Neural Network based Deep Learning Framework for Detection of Obstructive Sleep Apnea Syndrome}

This disease also can lead to high rates of employment and road accidents, among other effects.

Not only that, OSAS can affect children as well. This was shown when [4] surveyed that there were $1-5.7 \%$ from new-borns to young people who were living with OSAS. Despite that, OSAS has been widespread in pre-schoolers aged three to five, since there are high peaking of lymphoid tissue growth. OSAS can affect their daily lives since they are still small and kids and their will make a joke of them if OSAS children sleep during classes.

\section{THE CONCEPT OF MEDICAL INFORMATICS}

Study and application of methods for strengthening patient data collection, population data, diagnostic records and other knowledge related to patient treatment and public safety have been researched and used in medical informatics. It is a young science that evolved in the decades after the digital computer was invented in the 1940s. [7] identified that medical technology has a multidisciplinary dimension which interacts with various fields, including public health, biomedical science and computer science, cognitive sciences and information. By the 1970s, medical information engineering had invaded the medical world. Doctor's offices, hospitals and even small clinics all used computer-based medical reports to help track patient treatment and improvement.

Two hundred years ago, [8] health workers established scientific data gathering and storage as a means for collecting data. Nevertheless, few doctors knew that the manner in which illness and its diagnosis were documented had to alter empiricism by more ritualistic and testable methods. In this new area, however, the medical and organizational structures that affect random choice in therapies must be substituted by therapy that both be formalized, evaluated and fairly utilized. That is due to the fact this modern medicine has moved away from seeing a classification of illnesses in order to make it more comprehensible to the illness. By looking things at a system level, hospitals or even the patient himself will become more and more acquainted with what it is to be ill and how it can be described or reversed.

Medicine and medical care is rapidly driving the economy around the world $[14,15]$ and IT is one of their most valuable resources [13]. Effective and efficient information systems are therefore particularly needed. Such structures should, moreover, be constantly adjusted to the changing demands in medicine and medical care.

\section{DEEP LEARNING ALGORITHM}

Deep learning, which has compromised several hidden layers of artificial networks has become evident in Machine Learning (ML) research since 2016. The best efforts of the Artificial Intelligence (AI) community for a long time have made deep learning significant progress in solving problems, because deep learning appears to be the best way in high-dimensional information to recognise structures in high-dimensional data thus, to be important for many areas of business, research and government. In addition, Deep Learning has also pushed through other techniques of ML for predicting prospective drug molecules, brain circuit reconstruction, analysing particle accelerator data and forecasting the effects on disease and expression of gene mutations in DNA non-coding. It has also provided outstanding results in the field of Natural Language processing for various tasks, especially in the areas of sentiment analysis, language translation and the response of questions [9].

In previous literature, [1] used two hybrid models which are convolutional neural network (CNN) and recurrent neural network (RNN) to detect vital parameters and Deep Learning Algorithm for apnoea detection. CNN is most commonly applied to analysing visual imagery.

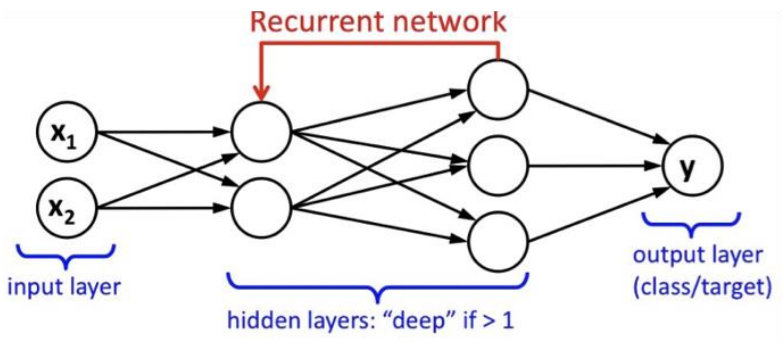

Figure 1. CNN image classification pipeline

Figure 1 shows the example of an architecture for CNN. CNN is most commonly applied to analysing visual imagery. CNN's information flow takes place in only one direction, passing the result from one input to another input [6]. Figure 2 shows the example of RNN. It is a class where there are connections between nodes from a directed graph along a sequence. RNN allows it to exhibit temporal dynamic behaviour for a time sequence.

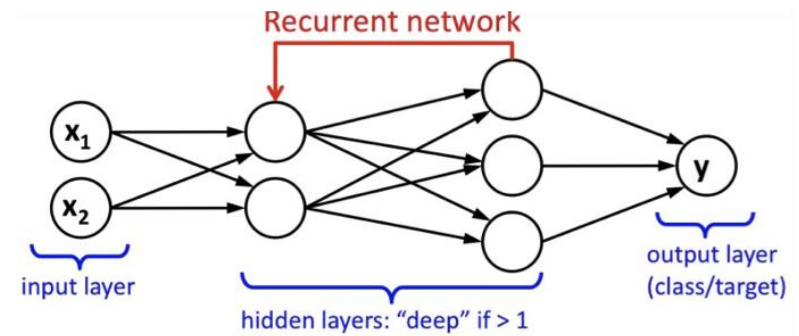

Figure 2. Example of RNN

Figure 2 shows a flowchart of the methods used in the paper. The trained network is used for the analysis and the capacity of the network to accurately identify the OSAS condition in the testing phase to interpret data details from segments other than those used for training. Therefore, the approach described in this article deletes the need for the use of independent extraction of features and OSAS classification algorithms.

\section{DATA ANALYSIS}

The proposed analysis consisted layered structures corresponding to CNN, where each layer has its clear-cut functions for supervised learning. The main standard of deep learning is to find new features that are well related to the small number of features that are available from the data in the training data set. Single lead ECG waveform recordings are used from several subjects with various OSAS conditions. Each wave recorded is considered to consists of several tandem sequential segments, lasting one minute each. Each segment is identified and analysed by medical experts to decide whether or not the segment constitutes an apnoeic case. 
Such waveform segments from the subjects form the input dataset and are fed into the deep learning algorithm based on the CNN. The various features are learned from the training data in different initial layers of the network classify there features into correct categories, such as normal/apnoeic episodes.

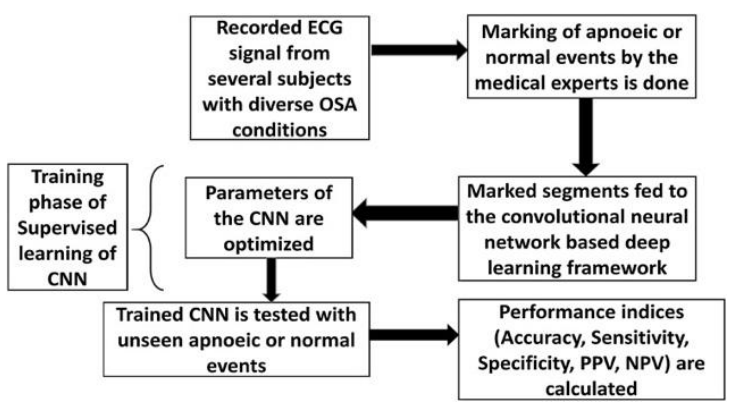

Figure 3. Flowchart of the proposed methodology

Figure 3 shows a flowchart of the methods used in the paper. The trained network is used for the analysis and the capacity of the network to accurately identify the OSAS condition in the testing phase to interpret data details from segments other than those used for training. Therefore, the approach described in this article deletes the need for the use of independent extraction of features and OSAS classification algorithms.

$\backslash$ Such network models have been used for image analysis for the majority of applications. In the work presented here, it is used to process time series data. In the following paragraphs, the planned network is discussed, and the complete scheme is shown in Figure 4. This is evident in Figure 4, which compromises of several layers in the CNN structures. These layers can be repeated $n$ number of times, depending upon the level of accuracy required. The layers are defined as follows:

\section{A. Input Layer}

The recorded lead ECG signals from different sleep-disorder subjects are received in this layer. The dimensions of different layers are shown in Figure 2. For each one-minute segment, each input data has 600 specimens.

\section{B. Convolution Layer}

$$
\boldsymbol{c}[\boldsymbol{n}]=s[n] * h[n]=\sum_{k=-\infty}^{k=+\infty} s[k] h[n-k]
$$

Each

layer conducts the 'convolution filtering' of the input data to determine the important features of the data and spreads information through the following layers. The 1D signal (sequence) convolution can be represented mathematically as where

$$
s[n]
$$

is the signal and

$$
h[n]
$$

is the convolutional kernel.

In the CNN, each convolutional represents a kind of functions, such as temporal variance design, flat data edges, subtle amplitude variations and many more. 20 convolution kernels have been used and kernel weights randomly initialized in the network listed. By back-propagation learning, weights are then modified. The dimension of the filter structure in the first convolution layer can be defined as [1 x $6 \times 20$ ]. It is done by test and error method, just as in the traditional Neural Network layer, the number of neurons is chosen.

\section{ReLU Layer}

It is the abbreviation of the Rectified Linear Units. This layer is based on a nonlinear decision- mapping function for a thresholding operation. The function in this work is

$$
\max \{0, s\}= \begin{cases}0 & \text { if } s<0 \\ s & \text { otherwise }\end{cases}
$$

\section{Pooling Layer}

The task of this layer is dimension reduction. For such an operation, max pooling is a very popular technique. Both average pooling and L2-norm pooling are in use. When the outputs are images, these pooling methods work very well. A modification is implemented by replacing the basic pooling operation by convolutional pooling, to fit the $1 \mathrm{D}$ output signals in the time domain. It is achieved by just entering a new convolution layer, but with a different slide along the time axis during kernel shifting. This is another novelty of the current scheme.

\section{Fully-Connected Layer}

Each neuron in the current layer is linked to all the activations of the previous layer. This layer is identical to conventional Neural Networks and as shown by its name. each layer is simply classifying, whereas the prior layers conduct operational learning. It should be remembered. For the network updated, the gradient-based back propagation algorithm is used. However, any other update algorithm can be chosen as defined by its specific problem.

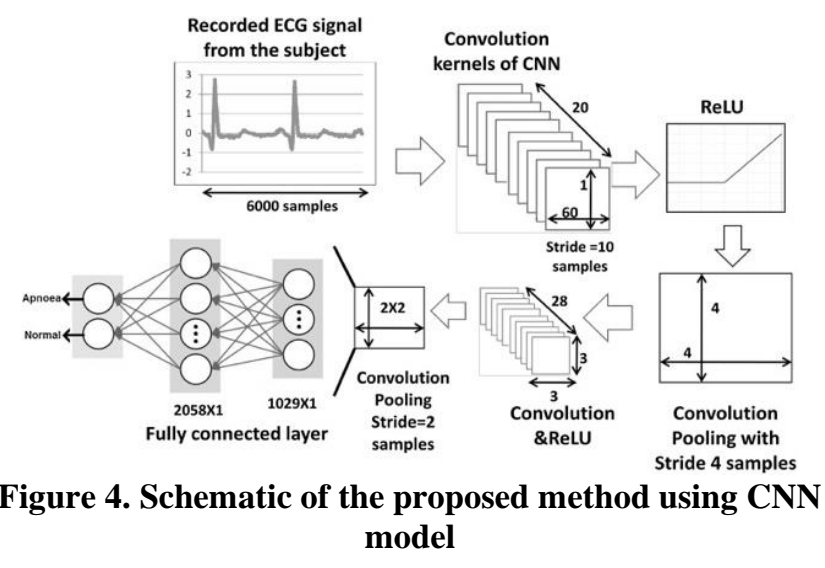

In the present work, the layers are repeated twice to achieve desired level of accuracy, which is shown in the Figure 4 . The CNN is trained using $2.3 \mathrm{GHz}$ Intel Core i5; RAM: $8 \mathrm{~GB}$ $2133 \mathrm{MHz}$ LPDDR3.

\section{RESULTS AND DISCUSSION}

The present system performance has been tested using the Physionet apnoea-ECG dataset [57]. Single lead ECG records were used with 35 subjects of OSAS. Table I shows the physiological parameters of the population under study. The processing time is 7-10 hours. As mentioned earlier, each information in the dataset is marked by experts' minute by minute. Such notes suggest whether sleep apnoea is active or not during the period. 


\section{Hybrid Bayesian Network in Neural Network based Deep Learning Framework for Detection of Obstructive Sleep Apnea Syndrome}

Each one- minute record can be assumed as 'one episode' or an 'event'. Accuracy, sensitivity and specificity of the proposed scheme are assessed. Since these parameters are well known, their expressions are not given.

The network is used for training by $50 \%$ of the available data and the rest is used for testing. The performance of the proposed network offers a scoring from 0 to 1 to indicate if the ECG episode is normal or an apnoeic event. At the training phase, an apnoeic occurrence was set as $\left[\begin{array}{l}1 \\ 0\end{array}\right]$ and a normal breathing event was measured as

Therefore, the network should be $\left.\left[\begin{array}{l}1 \\ 0\end{array}\right]_{\text {for }} \quad \begin{array}{l}0 \\ 1\end{array}\right]$ an apnoeic episode and ${ }_{0}^{0}$ for a normal breathing event under ideal network $\quad\left[\begin{array}{l}0 \\ 1\end{array}\right]$ performance after training. The outputs are not ideal during practical testing. For $[0.89]$

instance, the output often comes in $[0.12]$ in an apnoeic event. Therefore, the output limit is used in the testing phase, making the two output elements equal to 1 and the other equal to 0 greater than the output matrix.

Table I: Physiological parameters of the population

\begin{tabular}{|c|c|c|c|c|c|c|}
\hline $\begin{array}{l}\text { Sub. } \\
\text { No. }\end{array}$ & $\begin{array}{c}\text { Length } \\
\text { in } \\
\text { minutes }\end{array}$ & $\begin{array}{l}\text { Apnoea } \\
\text { minutes }\end{array}$ & $\begin{array}{c}\text { Non- } \\
\text { apnoea } \\
\text { minutes }\end{array}$ & AHI & Age & Sex \\
\hline 01 & 484 & 0 & 484 & 0 & 31 & M \\
\hline 02 & 476 & 4 & 472 & 0.38 & 30 & $\mathrm{~F}$ \\
\hline 03 & 474 & 3 & 471 & 0.13 & 28 & $\mathrm{~F}$ \\
\hline 04 & 539 & 425 & 114 & 71.8 & 29 & $\mathrm{~F}$ \\
\hline 05 & 558 & 516 & 42 & 93.5 & 29 & $\mathrm{~F}$ \\
\hline 06 & 471 & 0 & 471 & 0 & 41 & $F$ \\
\hline 07 & 496 & 434 & 62 & 75 & 60 & $\mathrm{M}$ \\
\hline 08 & 499 & 588 & 11 & 75 & 60 & M \\
\hline 09 & 521 & 344 & 177 & 15.1 & 57 & M \\
\hline 10 & 511 & 291 & 220 & 48 & 55 & M \\
\hline 11 & 430 & 1 & 429 & 0 & 31 & M \\
\hline 12 & 528 & 119 & 409 & 14.3 & 43 & $\mathrm{M}$ \\
\hline 13 & 483 & 2 & 481 & 0 & 27 & $\mathrm{~F}$ \\
\hline 14 & 511 & 120 & 391 & 19 & 53 & M \\
\hline 15 & 524 & 375 & 149 & 63 & 44 & M \\
\hline 16 & 511 & 120 & 391 & 19 & 53 & M \\
\hline 17 & 514 & 264 & 250 & 43 & 51 & M \\
\hline 18 & 488 & 407 & 81 & 56.2 & 54 & M \\
\hline 19 & 460 & 2 & 458 & 0 & 27 & $\mathrm{M}$ \\
\hline 20 & 401 & 1 & 400 & 0 & 27 & $\mathrm{~F}$ \\
\hline 21 & 516 & 65 & 451 & 24 & 53 & $\mathrm{M}$ \\
\hline 22 & 499 & 200 & 299 & 15.9 & 63 & M \\
\hline 23 & 491 & 439 & 52 & 79.5 & 38 & M \\
\hline 24 & 507 & 292 & 215 & 18.7 & 57 & M \\
\hline 25 & 528 & 57 & 471 & 33 & 40 & M \\
\hline 26 & 458 & 13 & 445 & 5 & 52 & M \\
\hline 27 & 511 & 96 & 415 & 10 & 39 & $\mathrm{~F}$ \\
\hline 28 & 509 & 167 & 342 & 18.5 & 43 & M \\
\hline 29 & 518 & 324 & 194 & 48 & 55 & M \\
\hline 30 & 510 & 240 & 270 & 21 & 58 & M \\
\hline 31 & 451 & 0 & 451 & 0 & 31 & M \\
\hline 32 & 506 & 316 & 190 & 34 & 55 & M \\
\hline 33 & 483 & 0 & 483 & 0 & 39 & M \\
\hline 34 & 466 & 12 & 454 & 0.13 & 44 & $\mathrm{~F}$ \\
\hline 35 & 470 & 209 & 261 & 37.7 & 46 & M \\
\hline Mean & 494.34 & 184.2 & 314.1 & 26.8 & \begin{tabular}{|l|l|}
44.1 \\
\end{tabular} & \\
\hline
\end{tabular}

Table II shows the results of the new system as well as the correlation of its output with current methods. All quality parameters for the proposed scheme are clearly very high in comparison to those of existing methods when evaluated on the same data set and indicate that the proposed technique has a positive contribution. Table III provides a detailed view of the performance of the current scheme.

Table II. Comparison of performance of the proposed scheme with existing approaches $(50 \%$ of training data and $50 \%$ of testing data)

\begin{tabular}{|l|l|l|l|}
\hline \multicolumn{1}{|c|}{ Methods } & \multicolumn{1}{c|}{ Accuracy } & \multicolumn{1}{c|}{ Specify } & Sensitivity \\
\hline Varon et al. [10] & 84.74 & 84.69 & 84.71 \\
\hline Hassan [5] & 87.33 & 90.72 & 81.99 \\
\hline Hassan and Haque & 85.97 & 86.83 & 84.14 \\
\hline
\end{tabular}

\begin{tabular}{|l|l|l|l|}
\hline$[11]$ & & & \\
\hline Proposed method & 98.91 & 99.20 & 97.82 \\
\hline
\end{tabular}

Table III. Confusion matrix and performance indices of the proposed scheme

\begin{tabular}{|c|l|l|}
\hline Results & \multicolumn{1}{|c|}{ Truth Apnoea } & \multicolumn{1}{c|}{ Non-apnoea } \\
\hline Apnoea & True Positive (TP) & False positive (FP) \\
& 4878 & 46 \\
\hline Non-Apnoea & False Negative (FN) & True Negative (TN) \\
& 109 & 5754 \\
\hline Total & 4987 & 5800 \\
\hline
\end{tabular}

$$
\begin{aligned}
& \text { Accuracy }=\frac{T P+T N}{T P+T N+F P+F N}=98.91 \\
& \text { Sensitivity }=\frac{T P}{T P+F N}=97.82 \\
& \text { Specificity }=\frac{T N}{T N+F P}=99.20 \\
& \mathrm{PPV}=\frac{T P}{T P+F P}=99.06 \\
& \mathrm{NPV}=\frac{T N}{T N+F N}=98.14
\end{aligned}
$$

\section{CONCLUSION}

In order to extend the performance and models of Bayesian Networks to medical care, rigorous studies on medical workflow and. On the perception of the algorithm of Deep Learning will be essential, based on the existing analysis. The study has been done. In summary, this study focused on the creation of convolutional neural network using Deep learning framework for the diagnosis of OSAS, with the aim of making the medical workflow for patients clearly and holistically. As stated in the literature review, the research study was endorsed in all the literature on Obstructive Sleep Apnoea, the concept of Medical Informatics, and Neural network.

This paper offers a deep learning framework utilizing CNN to simplify OSAS using single ECG signals. Improved OSAS identification precision in comparison to the current approaches is the main contribution of the research. The present scheme reduces the need to isolate and identify different functions algorithms. A section of the proposed network carries out characteristics and the other component classifies them in a controlled way. It has been found that the present method improves the classification accuracy on an average by a margin of more than $9 \%$ compared with existing methods. With less training data, the proposed scheme will learn inherent features and can also to some degree remove the impact of alterations in the data.

The downside of the scheme is its high computational burden, as it uses a profound deep learning approach. As the signal records for OSAS are lengthy (7-10 h) and the expert testing process is mostly offline, this restriction in its actual application does not hinder. However, the measurement strain is not as a consequence of the microcontroller's implementation of the algorithm, because deep learning frameworks are already deployed with ARM microcontrollers. 
The software module for applying the whole algorithm mentioned in the study may therefore be a good candidate to combine with a portable health monitoring device and since it operates on ECG signals, there is no need for a separate PSG program. The system is, however, a common one that be used for the same function for other physiological stimuli such as electroencephalogram (EEG), electrooculography (EOG), electromyography (EMG).

More studies are required to better fit a model of support for clinical decisions in to clinical practice, particularly when looking forward to support primary care, but this study clarified the need to incorporate a far broader set of clinical variables into a diagnostic model for OSAS. But nonetheless strengthened the advantages of CNN models for the task.

\section{REFERENCES}

1. Pomprapa, A., Sayani, S. M., Anwar, T., Stollenwerk, A., Kowalewski, S., \& Leonhardt, S. (n.d.). Apnea Detection in a Contactless Multisensor System using Deep Learning Algorithm.

2. Stone, J. P. (2002). Obstructive sleep apnoea. Lancet, 360(9350), 2078-2079. https://doi.org/S0140- 6736(02)11964-7 [pii] $\backslash r 10.1016 / S 0140-6736(02) 11964-7$

3. Rascu, A., Arhir, O. C., \& Otelea, M. (2016). Obstructive sleep apneaCase report and literature review. Romanian Journal of Legal Medicine, 24(2), 118-121. https://doi.org/10.4323/rjlm.2016.118

4. Da Silva Gusmão Cardoso, T., Pompéia, S., \& Miranda, M. C. (2018). Cognitive and behavioral effects of obstructive sleep apnea syndrome in children: a systematic literature review. Sleep Medicine, 46, 46-55. https://doi.org/10.1016/j.sleep.2017.12.020

5. Hassan AR. Computer-aided obstructive sleep apnoea detection using normal inverse Gaussian parameters and adaptive boosting. Biomed Signal Proc Control. 2016;29:22-30. doi: 10.1016/j.bspc.2016.05.009.

6. Rawat, W., \& Wang, Z. (2017). Deep Convolutional Neural Networks for Image Classi cation: A Comprehensive Review.

7. Wyatt, J. C. (2002). Basic concepts in medical informatics. Journal of Epidemiology \& Community Health, 56(11), 808- 812 https://doi.org/10.1136/jech.56.11.808

8. Coiera, E. (1998). Medical informatics meets medical education. Medical Journal of Australia, 168(7), 319-320. https://doi.org/10.1001/jama.273.13.1061

9. Xie, Y ., Le, L., Zhou, Y ., \& Raghavan, V . V . (2018). Deep Learning for Natural Language Processing. https://doi.org/10.1016/bs.host.2018.05.001

10. Varon C, Caicedo A, Testelmans D, et al. A novel algorithm for the automatic detection of sleep apnea from single-lead ECG. IEEE Trans Biomed Eng. 2015;62(9):2269-2278. doi: 10.1109/TBME.2015.2422378.

11. Hassan AR, Haque MA. Computer-aided obstructive sleep apnea screening from single-lead electrocardiogram using statistical and spectral features and bootstrap aggregating. Biocybern Biomed Eng. 2016;36(1):256-266. doi: 10.1016/j.bbe.2015.11.003.

12. Maspero, C., Giannini, L., Galbiati, G., Rosso, G., \& Farronato, G. (2015). Obstructive sleep apnea syndrome: a literature review. Minerva Stomatologica, 64(2), 97-109. Retrieved from http://www.ncbi.nlm.nih.gov/pubmed/25747430

13. Ammenwerth E, Haux R, Kulikowski C, Bohne A, Brandner R, Brigl B, Fischer G, Garde S, Knaup P, Ruderich F, Schubert R, Singer R, Wolff AC. Medical informatics and the quality of health: new approaches to support patient care - findings from the IMIA Yearbook of Medical Informatics 2003. Methods Inf Med 2003; 42 (2): 185-189.

14. Haux R, Ammenwerth E, Herzog W, Knaup P. Health care in the information society. A prognosis for the year 2013. Int J Med Inf 2002; 66 (1-3): 3-21. doi: 10.1016/S1386- 5056(02)00030-8.

15. Nefiodow LA. Der Sechste Kondratieff - Wege zur Produktivität und V ollbeschäftigung im Zeitalter der Information. Sankt Augustin: RheinSiegVerlag; 2001.

\section{AUTHORS PROFILE}

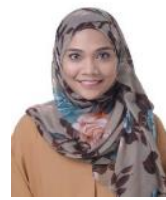

Farah Nadiah Binti Mohamed Farouk is a second yea Master student and a full-time General Assistant in the Computer Sciences Department at Universiti Teknologi PETRONAS (UTP), Perak Malaysia. Her main research interests are neural network, deep learning algorithms, and artificial intelligence. Her research concerns are also inclined towards the medical aspects as she is currently doing an implementation of a neural network in deep learning framework for Obstructive Sleep Apnea Syndrome for her Master thesis. She obtained her Bachelor's Degree in Information Technology with major of Software Engineering and Corporate Manager (minor) at Universiti Teknologi Petronas, Perak Malaysia where she has developed a mobile application for the medical field as her final year project.

In May 2017, Farah also has successfully passed the Certified Professional Requirement Engineering (Foundation Level) exam under International Requirements Engineering Board (IREB). She also did her internship for 8months in 2016 with Intel Technology Sdn. Bhd. Kedah, Malaysia where her project was to design a streamline as well as systematic system in processing request tickets for MSD staffs to look up, do the weekly report, and calculate the graph more precisely. This is to ensure accurate and precise information being captured and the quality is not being compromised and adherence to the ticketing process.

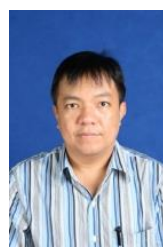

Toni Anwar graduated at RWTH Aachen University, Germany as Dr. rer. nat. in the area of Mobile Communications and studied International Management at TU Berlin, Germany. He is an Assoc. Prof. Dr. at Universit Teknologi Petronas and worked for multinational companies (Ericsson Sweden, Motorola USA, Siemens Germany etc.) for more than 10 (ten) years and in academic world for more than 15 (fifteen) years in different countries such as Germany, Thailand, Malaysia and Indonesia. He is an IEEE Senior Member and also MBOT professional member as Professional Technologies. His research areas are Software Engineering, Embedded System/IoT, Smart Agriculture, Smart Cities, Artificial Intelligence, Data Analytics, Location Based Services, Augmented Reality, Connected Healthcare and ICT. His research grants include international grants from Europe, Japan, Thailand, Malaysia, Indonesia from industry, academic and government related organizations.

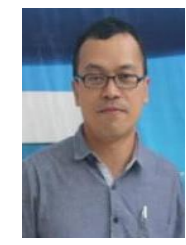

Nordin Zakaria is a senior lecturer with the Computer and Information Science Department, Universiti Teknologi PETRONAS (UTP) Malaysia. He has been with UTP for more than 10 years. Prior to that, he was a researcher in the Max-Planck-Institut fur Informatik, Germany, and a lecturer in Universiti Putra Malaysia. Dr Nordin Zakaria earned his PhD in Computer Science from Universiti Sains Malaysia, working in the area of Computer Graphics. His interest over the years has been diverse, ranging from user interface design to computational engineering. The main theme in his research has however been always metaheuristics methods, especially genetic algorithm, dealing with various computational problems in various domains. 